\title{
Metro Passenger Flow Forecasting Using 2A-LSTM with Multi-source Data
}

\author{
Shun $\mathrm{Yu}^{1}$, Xiaoli Zhi ${ }^{1+}$ and Weiqin Tong ${ }^{1}$ \\ ${ }^{1}$ School of Computer Engineering and Science, Shanghai University, Shanghai, China
}

\begin{abstract}
Short-term passenger flow forecasting has great significance for the safety and efficiency of urban rail transit system. The existing forecasting methods mostly focus on traffic data and consider little other external factors from multi-source data such as meteorological data and point of interest data, which may exert a strong influence on the passenger flow. This paper proposes a new type of LSTM with two attention mechanisms, named as 2A-LSTM, with dataset of multivariate time series to improve the forecasting accuracy. The multivariate time series are constructed from the multi-source data by means of data fusion. These time series have characteristics of strong self-correlation, periodicity and predictability, which are the key to ensure the prediction accuracy of 2A-LSTM. The 2A-LSTM uses temporal pattern attention and soft attention mechanism to perceive the correlation of each external factor on previous time steps. Based on Shanghai Metro traffic card data, we perform experiments to measure and compare the effect of external features on the accuracy of passenger flow by using different features combination. We input time series data of single station to our model and forecast the inbound passenger flow on weekdays. The experiment results show that the accuracy is improved by using external features, and our model has good performance on multivariate time series forecasting.
\end{abstract}

Keywords: short-term passenger flow forecasting, multi-source data fusion, attention mechanism, LSTM

\section{Introduction}

With the continuous development of urbanization in China, the urban rail transit system has been rapidly developed. Urban rail transit has the advantages of large traffic volume, low pollution, energy saving, and it is fast, convenient, safe and comfortable as well. At present, in many large and medium-sized cities, such as Beijing, Shanghai and Guangzhou, the average daily passenger flow of the metro has reached up to ten million or higher.

Taking Shanghai Metro as an example, by 2019, there were 15 metro lines, with a total length of 669.5 kilometres and an average daily passenger flow of nearly 11 million. For such a complex metro network and huge passenger flow, it is particularly important to mine the pattern of passenger flow and understand passenger demand. Metro operators demand an accurate prediction of real-time passenger flow to improve operation management and ensure safe operation in a timely manner. Passengers need flow forecasting to adjust their travel plans dynamically.

The metro widely adopts the Automatic Fare Collection Systems (AFCs), which keep the departure, destination and time information for each passenger trip. AFCs generates massive amounts of data every day. The AFC data makes it possible to understand the spatiotemporal distribution regularity of passenger travel. Moreover, due to the progress of data acquisition technology, rich, accurate and timely external data affecting the passenger flow from different sources, such as meteorological observation, point of interests (POI), Weibo news, become available. Thus, prediction of metro passenger flow with multi-source data is necessary and practicable.

The contributions of this paper are as follows:

(1) A dataset of multivariate time series is built for short-term passenger flow forecasting. This dataset is collected from multi-source data by means of data fusion and expansion. The time series are

\footnotetext{
+ Corresponding author. Tel.: + 13918483274.

E-mail address: xlzhi@shu.edu.cn.
} 
sampled on a recommended time granularity. Our dataset fits the passenger flow patterns of specific/different dates and stations, and is effective to train our flow forecasting model.

(2) 2A-LSTM (long short-time memory neural network with two attention mechanisms) is proposed for multivariate time series forecasting. This model uses temporal pattern attention and soft attention mechanism for capturing the relevance between external factors and past flow on multivariate time series. Thus, the influence of external factors and historical passenger flow pattern can be better learned to forecast future passenger flow.

\section{Related Work}

The main research goal of this paper is to realize the short-term passenger flow forecast of urban rail transit network. In the past decade, researchers have done a lot of research and contributed a wealth of passenger flow prediction models and methods.

At present, the research on short-term passenger flow forecast of Metro can be divided into the following three categories: (1) time series forecasting model based on statistical theory; (2) passenger flow forecasting method based on machine learning; (3) passenger flow forecasting method based on and deep learning technology.

Time series model is a kind of statistical model, it is a common prediction method and widely used in the transportation domain. Time series forecasting model focuses on the change of samples with time, which is generally divided into trend change, periodic (seasonal) change, non-stationary (random) change.

Zhu et al. [1] analysed the change of Shanghai metro passenger flow and established the ARIMA (Autoregressive Integrated Moving Average) model of Shanghai metro daily passenger flow forecast, which has a good performance for the large passenger flow forecast before and after the main holidays. Guo et al. [2] considered that the traditional time series models overlooked the short-term passenger flow volatility. They combined SARIMA (Seasonal Autoregressive Integrated Moving Average) with GARCH (Generalized Autoregressive Conditional Heteroscedasticity) model to study the interval prediction of traffic passenger flow. It makes up for the blank of traffic flow level prediction and associated prediction interval.

The machine learning methods, thanks to their stronger adaptability self-organization and self-learning ability, can yield performance in the simulation of passenger flow features.

Ding et al. [3] believed that current short-term passenger flow forecasting method lacks consideration of bus transfer and temporal features. A relatively novel data mining method, GBDT (Gradient Boosting Decision Tree), is applied to the short-term passenger flow forecasting of subway to capture the association between independent variables. Based on the traffic smart card data of three subway stations in Beijing, they established GBDT models with different parameters to verify the effectiveness of the model. Liu et al. [4] established a series of passenger flow forecasting models with different input features by using random forest method. The experimental results show that the model has achieved good results for the short-term passenger flow forecast of weekdays, weekends and holidays.

Deep learning technology, represented by deep neural network, has been widely used in the field of transportation. Sha et al. [5] combined the Shanghai Metro station passenger volume with meteorological data, and used the dataset of each period in three different models for rolling forecasting from 15 minutes to 6 hours: LSTM (Long Short-term Memory), GRU (Gated Recurrent Unit) and BPN (Back Propagation Network). The results showed that these models were feasible and effective in short-term passenger flow forecasting. Han et al. [6] proposed a new method based STGCNN (spatiotemporal graph convolutional neural networks), which transforms urban subway network into graph and uses graph convolutional neural network to forecast. The performance of their model for forecasting the short-term passenger flow of Shanghai urban rail transit network was better than other commonly used models.

By analysing the correlation between passenger flow distribution pattern and external influence factors, we can realize more effective short-term passenger flow forecasting.

Tang [7] and Wang [8] have studied the influence of external weather factors on passenger flow forecasting successively. They analysed the correlation between weather factors (such as weather conditions, air quality and temperature) and passenger travel. From the case analysis of urban rail transit datasets in 
Shenzhen and Beijing, the results showed that adding meteorological data to passenger flow forecasting model can help to improve the prediction accuracy. Zhu et al. [9] took Ningbo City as an example, and statistically analysed the correlation among the station passenger flow and the nature of land and the distribution of buildings. Zhao et al. [10] used the decomposition theory to analyse the impact of large-scale special activities on the surrounding subway stations. They established an estimation model according to the preference survey data. Based on this model, the passenger flow of the station during actual certain special events around Beijing subway station was predicted, which verified the practicability of the method and achieved good prediction results. Chen et al. [11] considered the dynamic fluctuation and non-linearity of passenger flow during special events. They built a regression model based on the traffic card data of Nanjing station, which effectively captured the mean value and fluctuation of passenger flow during special events. This model is superior to traditional methods in accuracy and reliability.

By analysing the correlation between passenger flow distribution pattern and external influence factors, we can realize more effective short-term passenger flow forecasting. And the existing research proved the correlation of some factors like weather and so on with passenger flow. Thus, we collected various external factors data and validate the effectiveness of them. Based on 2A-LSTM, the prediction method using external factors can achieve higher prediction accuracy.

\section{Methodology}

\subsection{Problem Definition}

This paper mainly focuses on the short-term passenger flow forecasting of single station. It can be expressed as Equation (1):

$$
f_{t}^{s}=\operatorname{pred}\left(\left[\begin{array}{cccc}
f_{t-1}^{s} & f_{t-2}^{s} & \cdots & f_{t-k}^{s} \\
\boldsymbol{E F}_{t-1}^{s} & \boldsymbol{E} \boldsymbol{F}_{t-2}^{s} & \cdots & \boldsymbol{E F}_{t-k}^{s}
\end{array}\right]\right)
$$

where: $f_{t}^{s}$ refers to the $s$ station's passenger flow at the $t$ moment, $\boldsymbol{E} \boldsymbol{F}_{t}^{s}$ refers external features of $s$ station on $t^{\text {th }}$ time step from multivariate data, and $k$ is the maximum number of time steps to input. In the rest of this paper, we will discuss the selection of multivariate data and the prediction methods.

\subsection{Data Description and Pre-processing}

This paper uses multi-source data including the passenger flow dataset which was collected from the Shanghai public traffic card and three types of external data including the meteorological, POI (Point of Interest) and metro fault data. In order to be used for short-term passenger flow forecasting, the above datasets are pre-processed by a series of strategies:

(1) passenger flows are sampled according to given time granularity to obtain different time series of passenger flow;

(2) the weather features of the same period are obtained which includes temperature, humidity, wind speed, apparent temperature and weather conditions;

(3) the POI distribution rate of 800 meters around each metro station are calculated according to the stations' location and obtain POI features;

(4) metro fault information (fault datetime, duration and affected stations) is collected from official Weibo of Shanghai Metro and match with passenger flow data one by one;

(5) passenger flow trend and level of each time step are calculated to obtain passenger flow condition features condition features.

After above data pre-processing, we use hourly passenger flow of each days as ridership indicator, and run twice K-means cluster algorithm. The first clustering is used to clustering passenger flow series on different days of week by using ridership indicators of specific station. The second clustering is used to cluster different stations features by using ridership indicator and poi features of each stations.

Fig. 1 shows the hourly passenger flow of one specific station in a specific week (from April $13^{\text {rd }}$ to $19^{\text {th }}$, 2015), and the results of the first clustering result are indicated by different colours. Obviously, there are different passenger flow pattern in different days of the week. Our passenger flow forecasting task mainly focuses on the inbound passenger flow on weekdays in the rest of this paper. 

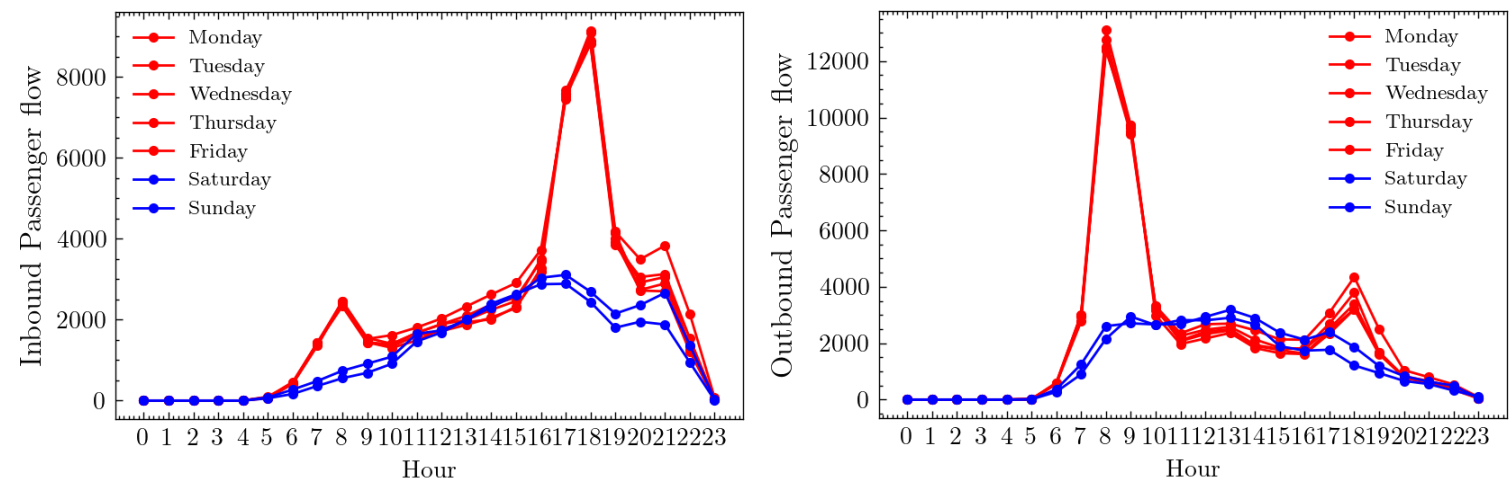

Fig. 1: Hourly Passenger flow of Line 2 Nanjing Rd.(W).

After the second clustering, 313 stations of Shanghai are divided into five categories i.e., residential stations, workplace stations, far residential stations, mixed residential stations, and mixed workplace stations. The rest of this paper will focus on the passenger flow of workplace stations represented by Nanjing West Road Station. And our forecasting method will be applied to other stations to verify its generality.

Besides, only the prediction of a stable time series is possible. Non-stationary time series is currently theoretically flawed, and its prediction methods and theory have limitations.

We used 16 time granularities (including 1-6 $\mathrm{min}, 810 \mathrm{~min}, 12 \mathrm{~min}, 15 \mathrm{~min}, 20 \mathrm{~min}, 30 \mathrm{~min}, 1-3$ hour) to sample our time series. And we assess the predictability of the sampled series at different time granularities by calculating their autocorrelation and doing ADF (Augmented Dickey-Fuller) test on these series on each of clusters. Assessment results are obtained by calculating the average value of clusters' results.

The significance thresholds of self-similarity and ADF tests were set to 0.95 and 0.01 , respectively. The assessment results of workplace stations' series are given as in Fig. 2, where the red dotted line is correlation coefficient threshold, and the red bar represents unqualified ADF test results. Through the stationarity test, the time series of $4-15 \mathrm{~min}$ is recommended. The next experiments in this paper will be based on the passenger flow sampled in 5 minutes. Finally, we do fusion of the features of passenger flow, passenger flow conditions, datetime, weather, poi, metro failure to the construct multivariate time series for forecasting.
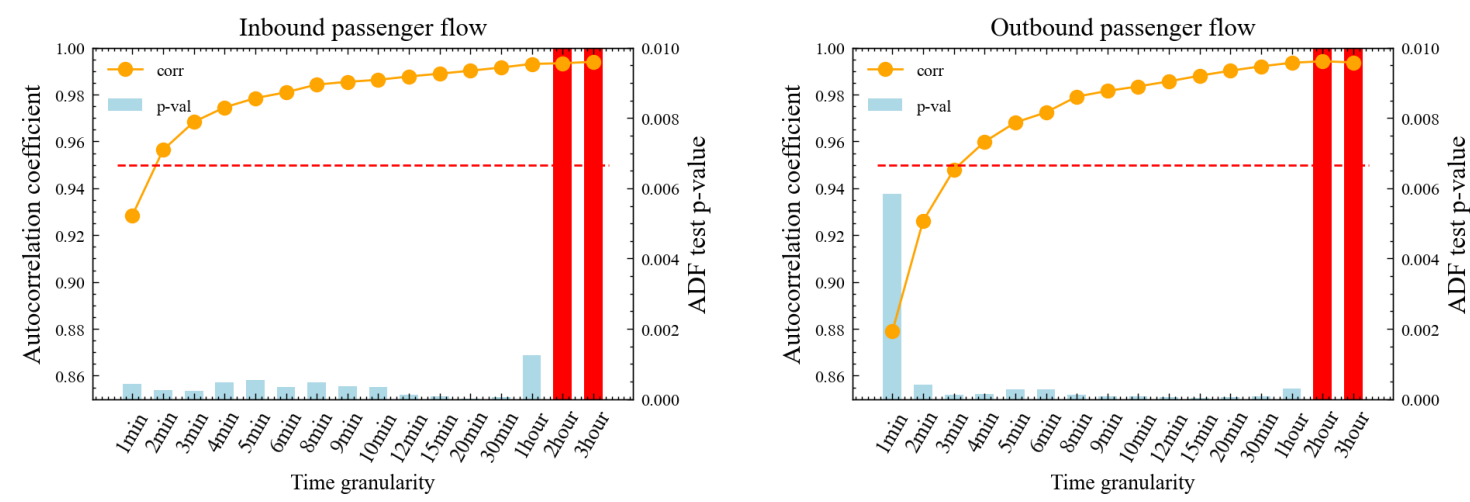

Fig. 2: Assessment results of workplace stations.

Overall, our original passenger flow data has over 300 million traffic card records, and we obtain the final dataset of 313 stations in 21 weekdays with 1893024 rows and 44 columns (passenger flow and other external features). And we divide this dataset into train set, verify set and test set, with a proportion of 5:1:1.

\subsection{A-LSTM}

We use a new 2ALSTM with two attention mechanisms, namely soft attention and temporal pattern attention, for passenger flow forecasting. The temporal pattern attention (TPA) [12] is mechanism to select the relevant time series instead of the time steps as the other attention mechanism does, so it enables model to learn more effectively the influence of different features on the predicted results. The soft attention is used to learn dependency of previous time steps. Fig. 3 shows the architecture of our model. The rest of this section will describe the detail of each layer. 
Input Layer. The input of the model is multivariate time series. This layer is first fully connected layer and used to features conversion so that input features are easier to learn and train.

LSTM Layer. LSTM layer is used to processing time series. Each of time steps' features are input to LSTM block cell one by one. It calculates the hidden states and output of each timestep as $H_{t}=$ $\left\{h_{t-w}, h_{t-w+1}, \cdots, h_{t-1}\right\}$ and $O_{t}=\left\{o_{t-w}, o_{t-w+1}, \cdots, o_{t-1}\right\}$, where $w$ is number of time step. $H_{t}$ and $O_{t}$ are input to two attention mechanisms, respectively.

Temporal Pattern Attention. TPA is used to choose different feature variables to weight because each feature contributes differently to the passenger flow. We use a convolution layer to capture various signal patterns by applying CNN filters on the row vectors of $\mathrm{H}$. And the sigmoid activation function is used to expect the weight of attention, which is convenient to select multiple variables.

Soft Attention. A typical attention mechanism to capture the influence of different time steps. It uses a scoring function to calculate the attention distribution of different inputs of $O$, finally, corrects the prediction results by weighted average.

Output Layer. This layer is used to fusion result. We integrate the results of two types of attention mechanisms. The final result is obtained through the second fully connected layer, and it is calculated through a clamp function to avoid getting meaningless negative value.

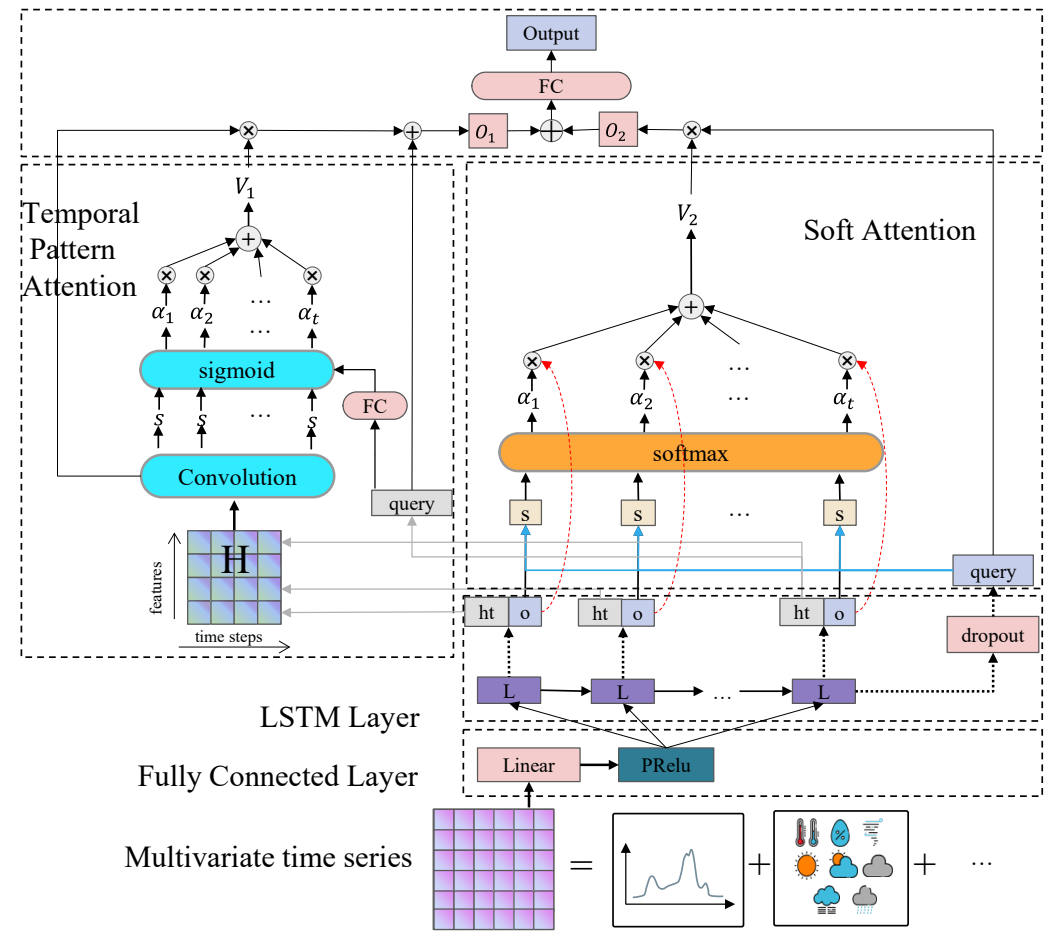

Fig. 3: Structure of 2A-LSTM

\section{Experiment}

\subsection{Experimental Setup}

We set Adagrad as model's optimization method, and use an adaptive learning rate (the initial learning rate is 0.05 , reduce factor is 0.5 ). The size of LSTM layers is 128 and the number of layers is 2 . The convolution layer of TPA has 1 input channel and 16 output channels with $(12 \times 1)$ kernel. The batch size for training dataset is set as 32, and the numbers of time steps are set as 6 and 12. Two indicators are used to evaluate our model's performance: Mean Absolute Error (MAE) and Root Mean Square Error (RMSE).

In order to validate the effectiveness of our dataset and model, we set up three groups of experiments:

(1) We use single LSTM network and ALSTM (LSTM with only soft attention mechanism) as baseline and input datasets of case 1 to 5 which are indicated in Table 1 .

(2) We input features of case 6 to our model and other multivariate time series prediction models: TPALSTM [12], LSTNet [13] and MQ-RNN [14] for comparison. 
(3) We train our model by using different workplace stations to verify the generality and use extra evaluating indicator MAPE (Mean Absolute Percentage Error). All the above models are developed by PyTorch, and all experiment are carried out on NVIDIA Quadro P6000 GPU.

Table 1: Features of predictors

\begin{tabular}{|l|l|}
\hline & Features \\
\hline Case 1 & Inbound station passenger flow (univariate) \\
\hline Case 2 & Case 1 and flow and pois of station \\
\hline Case 3 & Case 1 and weather at the same period \\
\hline Case 4 & Case 1, date, time and fault effect \\
\hline Case 5 & Case 1 and other passenger conditions \\
\hline Case 6 & All the above features \\
\hline
\end{tabular}

\subsection{Result and Analysis}

The results of baseline experiments are presented in Table 2. By merging different external features, the accuracy of passenger flow prediction is improved. Weather features have the most notable effect on the accuracy. It can be interpreted that the weather has the greatest influence on passenger flow.

Table 2: Results of baselines (with 6 and 12 time steps input)

\begin{tabular}{|c|c|c|c|c|c|c|c|c|c|c|c|}
\hline \multirow{2}{*}{$\begin{array}{c}\text { Time } \\
\text { steps }\end{array}$} & Case & \multicolumn{2}{|c|}{ Case 1 } & \multicolumn{2}{c|}{ Case 2 } & \multicolumn{2}{c|}{ Case 3 } & \multicolumn{2}{c|}{ Case 4 } & \multicolumn{2}{c|}{ Case 5} \\
\cline { 2 - 13 } & Model & $M A E$ & $R M S E$ & MAE & RMSE & MAE & RMSE & MAE & RMSE & MAE & $R M S E$ \\
\hline \multirow{2}{*}{6} & LSTM & 23.36 & 40.66 & 22.53 & 39.43 & 21.69 & 37.89 & 21.86 & 37.74 & 21.27 & 37.28 \\
\cline { 2 - 14 } & ALSTM & 22.80 & 40.80 & 22.14 & 40.32 & $\mathbf{2 0 . 9 5}$ & $\mathbf{3 6 . 6 1}$ & 22.09 & 39.21 & 20.96 & 38.28 \\
\hline \multirow{2}{*}{12} & LSTM & 23.28 & 40.67 & 22.80 & 39.09 & 21.14 & 36.40 & 21.94 & 37.74 & 21.71 & 36.80 \\
\cline { 2 - 11 } & ALSTM & 23.22 & 41.42 & 22.76 & 39.31 & $\mathbf{2 0 . 4 2}$ & $\mathbf{3 3 . 9 1}$ & 21.95 & 37.64 & 20.78 & 34.47 \\
\hline
\end{tabular}

We use multivariate time series with all the features to test different models. Table 3 shows the second group of experiments. We observe that adding attention mechanism on LSTM improves prediction accuracy. and 2A-LSTM significantly outperforms other models. Using two attention mechanisms can effectively capture the influence of different time steps and different variables on the prediction results, respectively. It proves that all components added are useful for LSTM layers.

Table 3: Comparison against time series prediction models (using case 6 dataset)

\begin{tabular}{|c|c|c|c|c|c|c|c|}
\hline Time steps & Model & LSTM & ALSTM & TPALSTM & 2A-LSTM & LSTNet & MQ-RNN \\
\hline \multirow{3}{*}{6} & MAE & 21.32 & 19.49 & 18.09 & $\mathbf{1 6 . 7 7}$ & 18.50 & 17.90 \\
\cline { 2 - 8 } & RMSE & 37.41 & 34.86 & 28.42 & $\mathbf{2 8 . 0 8}$ & 29.95 & 29.84 \\
\hline \multirow{3}{*}{12} & MAE & 20.58 & 19.77 & 18.17 & $\mathbf{1 6 . 6 4}$ & 18.80 & 17.51 \\
\cline { 2 - 8 } & RMSE & 34.72 & 32.45 & 28.76 & $\mathbf{2 5 . 9 8}$ & 29.97 & 28.16 \\
\hline
\end{tabular}

Finally, our model is trained and tested on 20 workplace stations separately. The results of 5 examples are presented in Table 4. 2A-LSTM can forecast the single-station's passenger flow with relatively high accuracy for Shanghai Metro.

Table 4: Experiment results of example workplace stations (using case 6 dataset with 12 time steps)

\begin{tabular}{|c|c|c|c|c|c|}
\hline Stations & Xujiahui & $\begin{array}{c}\text { Shanghai Railway } \\
\text { Station }\end{array}$ & $\begin{array}{c}\text { Zhongshan } \\
\text { Park }\end{array}$ & $\begin{array}{c}\text { Changshou } \\
\text { Rd. }\end{array}$ & $\begin{array}{c}\text { Jing'an } \\
\text { Temple }\end{array}$ \\
\hline Max & 1028 & 761 & 488 & 721 & 745 \\
\hline MAE & 19.18 & 28.63 & 11.78 & 12.04 & 11.77 \\
\hline MAPE & $12.50 \%$ & $14.78 \%$ & $12.46 \%$ & $10.96 \%$ & $14.20 \%$ \\
\hline RMSE & 30.18 & 43.80 & 17.98 & 18.40 & 19.57 \\
\hline
\end{tabular}

\section{Conclusion}

This paper focuses on the short-term passenger flow forecasting task of Shanghai Metro. We constructed multivariate time series based on multi-source data including AFC (Automatic Fare Collection Systems) data, 
meteorological data, POI (point of interests) data and so on. And we designed a 2A-LSTM network which use temporal pattern attention and soft attention mechanisms, so it can capture impact of external features and pervious time steps to improve prediction accuracy. We construct three groups to experiments to forecast single station's passenger flow to validate the effectiveness of our dataset and model. The results of experiments showed that the added external features effectively improve the prediction accuracy. Our model achieves better performance than other multivariate time series prediction model. These methods can provide reference for other passenger flow prediction work.

This paper is an initial research of passenger flow forecast based on multi-source data fusion. In this paper, an end-to-end neural network is used to realize short-term passenger flow forecasting of urban rail transit, but there are still several problems that need to be further studied. For example, the data of neighbour metro stations transfer stations and passenger flow on different days are not used in our model, and other external factors like special events, metro fares, new constructed lines are not considered.

\section{Acknowledgements}

This paper is supported by Shanghai Science and Technology Committee of Shanghai Municipality under grant No.19511121002 and Shanghai Engineering Research Center of Intelligent Computing System.

\section{References}

[1] H. Zhu. N days average volume based ARIMA forecasting model for Shanghai metro passenger flow. 2010 International Conference on Artificial Intelligence and Education. Hangzhou, China, 2010: 631-635.

[2] Guo J, Huang W, Williams B M. Adaptive Kalman filter approach for stochastic short-term traffic flow rate prediction and uncertainty quantification. Transportation Research Part C, 2014, 43: 50-64.

[3] C. Ding, D. Wang, X. Ma, H. Li. Predicting short-term subway ridership and prioritizing its influential factors using gradient boosting decision trees. Sustainability. 2016, 8(11): 1100.

[4] L. Liu, RC. Chen, Q. Zhao, et al. Applying a multistage of input feature combination to random forest for improving MRT passenger flow prediction. Journal of Ambient Intelligence and Humanized Computing volume. 2019 .10: 4515-4532.

[5] S. Sha et al. RNN-Based Subway Passenger Flow Rolling Prediction. in IEEE Access, 2020, 8: 15232-15240.

[6] Y Han, S Wang, Y Ren, C Wang, P Gao, G Chen. Predicting Station-Level Short-Term Passenger Flow in a Citywide Metro Network Using Spatiotemporal Graph Convolutional Neural Networks. ISPRS International Journal of Geo-Information. 2019; 8(6):243.

[7] L. Tang, Y. Zhao, J. Cabrera, J. Ma and K. L. Tsui. Forecasting Short-Term Passenger Flow: An Empirical Study on Shenzhen Metro. in IEEE Transactions on Intelligent Transportation Systems. 2019, 20(10): 3613-3622.

[8] J. Wang, B. Leng, J. Wu, H. Du and Z. Xiong. MetroEye: A Weather-Aware System for Real-Time Metro Passenger Flow Prediction. in IEEE Access. 2020, 8: 129813-129829.

[9] J. Zhu, F. Hong, Jie. Liu. Correlation Analysis Between the Land Use and the Demand of Urban Rail Transport. In Traffic \& Transportation. 2020, 36(01): 88-91. (in Chinese)

[10] L. Zhao, E.J. Yao, S.S. Liu, et al. Passenger Flow Analysis for the Surrounding Subway Stations of Large Special Event Site during Dissipation. 2017 6th International Conference on Energy, Environment and Sustainable Development. 2017: 273-277.

[11] E. Chen, Z. Ye, C. Wang, M. Xu. Subway Passenger Flow Prediction for Special Events Using Smart Card Data. in IEEE Transactions on Intelligent Transportation Systems. 2020, 21(3): 1109-1120.

[12] S. Y. Shih, F. K. Sun, H. Y. Lee. Temporal pattern attention for multivariate time series forecasting. Mach Learn. 2019, 108: 1421-1441.

[13] G. Lai, W. C. Chang, Y. Yang, et al. Modeling Long- and Short-Term Temporal Patterns with Deep Neural Networks. The 41st International ACM SIGIR Conference. ACM, Session 1C: Prediction. 2018: 95-104.

[14] R. Wen, K. Torkkola, B. Narayanaswamy. A Multi-Horizon Quantile Recurrent Forecaster. arXiv preprint arXiv: 1711.11053, 2018. 